\title{
An improved power network partition method based on response characteristics of reactive power sources
}

\author{
Bing-Ke Shi ${ }^{\dagger}$ and Bao-Zhu Liu \\ School of Electrical \& Electronic Engineering, \\ North China Electric Power University, \\ Beijing, 102206, China \\ ${ }^{\dagger}$ E-mail: shibingke_1993@163.com \\ www.ncepu.edu.cn \\ Shuang Zhang \\ Ningxia Electric Power Research Institute, \\ Ningxia Hui Autonomous Region \\ Yinchuan, 750002, China \\ E-mail:0zhangsh0nx@163.com
}

\begin{abstract}
Based on the response characteristics, reactive power sources are divided into fast sources and slow sources. According to the thought of reactive power source buses partition and load buses merging, this paper proposed a power network secondary partition method based on the response characteristics of reactive power sources. Firstly, calculating the electrical distance based on the sensitivity of reactive power source buses with both fast sources and slow sources. Secondly, partitioning the power network by a hierarchical clustering method, and then merging the load buses. If the system response time is long enough, the slow sources can be regarded as same as the fast sources, so all reactive power sources are treated as fast sources. The partition results of reactive power source buses and load buses can be obtained in the same way, and this process is the secondary partition. Lastly, this method is verified by the IEEE 39-bus system and case results show it can improve economy and reliability of power network.
\end{abstract}

Keywords: Response Characteristics; Sensitivity; Secondary Partition; Electrical Distance

\section{Introduction}

The concept of voltage hierarchical control is firstly proposed by the EDF, which is the voltage control scheme on the basis of pilot buses and control area. At present, many countries around the world adopt this kind of control method[1-3]. In recent years, Chinese researchers has also started the research on voltage hierarchical control and achieved good practical results.

When the hierarchical voltage control is used, how to divide the power network into several mutually decoupled areas is vital[4,5]. At present, most partition 
methods are based on the sensitivity between different buses within power network. On the basis of sensitivity, the electrical distance between buses can be calculated, and then using the different partition algorithms to partition the buses[6,7].

The purpose of partition is to strengthen the connection of internal buses within each area and reduce the coupling degree between areas. The principles of partition are shown as follows.

(1) The controlled bus should be assigned in the same area with reactive power source bus which is most sensitive to this bus.

(2) Each area should have sufficient reactive power capacity to control its voltage changes.

(3) The buses of same area should have the strong coupling. Otherwise, the buses of different areas should have weak coupling.

In [8], an expert knowledge based upward hierarchical classification method has been proposed. A concept of reactive power control space is proposed in [9]. And in [10], a nested decomposition has been used to realize partition.

In the traditional power network partition, the reactive power outputs of all sources are regarded as the equivalent power outputs and ignore the factor of response speed. So it will influence the accuracy of bus partition. This paper took the response characteristics of reactive power sources into account and then proposed the concept of secondary partition. Firstly, based on the sensitivity distance which is the ratio of reactive power and another reactive power, the partition result of reactive power sources can be obtained. And then merging load buses according to the control ability of each reactive power control area. Finally, some digital test results for an IEEE 39-bus system are presented and show that this method is more reasonable than other methods.

\section{Sensitivity and Electrical Distance}

\subsection{Sensitivity definition}

Sensitivity is the change degree of an electricity quantity when another electrical quantity appears unit change. Sensitivity is used to reflect the coupling degree between two buses, and it is also the basis of calculating the electrical distance. Now the related research mainly considers the electrical distance between the load buses which both are usually PQ buses, so the sensitivity of voltage to reactive power is used to represent the relationship between the load bus reactive changes and other load buses' changes of voltage amplitude. In this paper, the sensitivity of reactive power source was studied. Considering the reactive power bus is usually PV bus, sensitivity of reactive power to reactive power is proposed to represent influence degrees, which are reactive power changes of 
other sources when one reactive power source appears unit change. And the sensitivity is defined as Eq. (1).

$$
s_{i j}=\frac{\Delta Q_{j}}{\Delta Q_{i}}
$$

Where, $\Delta Q_{i}$ is reactive power change at reactive power source bus $i, \Delta Q_{j}$ is reactive power change corresponding to $\Delta Q_{i}$.

$\mathrm{N}-\mathrm{R}$ power flow correction equation is indicated as Eq. (2).

$$
\left[\begin{array}{l}
\Delta P \\
\Delta Q
\end{array}\right]=\left[\begin{array}{cc}
H & N \\
J & L
\end{array}\right]=\left[\begin{array}{c}
\Delta \theta \\
\Delta V
\end{array}\right]
$$

The reactive power output matrix $Q=\left[\begin{array}{llll}Q_{1} & Q_{2} & \ldots & Q_{n}\end{array}\right]^{T}$ is obtained after flow calculation, where $n$ indicates the number of reactive power source buses. Recursive method is adopted to solve the problem, specific steps are as follows:

Step (1) Convert the reactive source bus $i$, which is to be solved, from PV bus to PQ bus, the rest are still PV buses.

Step (2) $Q_{i}$ reduce a unit value, and then a new reactive power output matrix

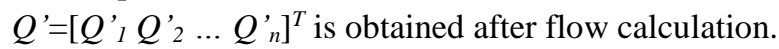

Step (3) Calculate the difference between $Q$ and $Q$, and then divided it by the reactive power unit value, the sensitivity between reactive power source $i$ and another reactive power source $j$ is obtained by Eq. (3).

$$
s_{i j}=\frac{\Delta Q_{j}}{\Delta Q_{i}}=\frac{Q_{j}^{\prime}-Q_{j}}{\Delta Q_{i}}
$$

Step(4) Repeat Step(1) to Step(3), until all the PV buses have been calculated.

According to the above steps, the sensitivity matrix of reactive power to reactive power $S_{G G}$ is shown in Eq. (4).

$$
S_{G G}=\left[\begin{array}{cccc}
s_{11} & s_{12} & \ldots & s_{1 n} \\
s_{21} & s_{22} & \ldots & s_{2 n} \\
\vdots & \vdots & \vdots & \vdots \\
s_{n 1} & s_{n 2} & \ldots & s_{n n}
\end{array}\right]_{n \times n}
$$

Where, $s_{i j}$ is a negative number.

\subsection{Electrical distance}

Based on the procedure mentioned before and method proposed in [9], electrical distance between bus $i$ and $j$ is defined in Eq. (5). 


$$
d_{i j}=-\lg \left|s_{i j}\right|
$$

Obviously, the less the value of $d_{i j}$ is, the more the sensitivity between bus $i$ and $j$ is, which means bus $i$ has a significant influence on bus $j$. Electrical distance matrix $D$ is formulated in Eq. (6).

$$
D=\left[\begin{array}{cccc}
d_{11} & d_{12} & \cdots & d_{1 n} \\
d_{21} & d_{21} & \cdots & d_{21} \\
\vdots & \vdots & \ddots & \vdots \\
d_{n 1} & d_{n 2} & \cdots & d_{n n}
\end{array}\right]
$$

\subsection{Load emergence}

After the partition of the reactive power source is determined, the load buses need to be merged into the corresponding reactive power source according to the shortest distance and the most sensitive principles. Considering the synthesized control capability of multiple reactive power sources to a single load, the sum of reactive power compensation in each partition's is defined as the ability to control the voltage of load buses after reactive unit fluctuation occur at load bus. Nevertheless, traditional load merging strategies may cause load buses merged in the shortest distant however poorest voltage control partition.

\subsection{Partition algorithm}

In [9], load bus partitioning method based on ward class distance and cohesion hierarchical clustering algorithm is proposed, where simulation analyzed the clustering effect of five class distances. It concludes that the combined effect of ward class distance is the best, and effective strategy to determine the number of partition using the platform area which occurred in clustering process is given. Reactive power source buses partitioning based on ward class distance and cohesion hierarchical clustering algorithm is also used in this paper. Use hierarchical clustering function linkage in Matlab software to partition

\section{Case Study}

First time threshold value $T$ is set according to the speed of response characteristics of reactive power source. The response rate of slow source is less than $T$ while the response rate of fast source is greater than $T$. Base on the principles mentioned above, the IEEE 39-bus test system is utilized to verify the proposed method. 


\subsection{Partition with slow sources}

The IEEE 39-bus test system comprises 10 reactive power source buses, assuming that the reactive power source bus 30, 34, 36 and 39 are slow sources. The rest are fast sources. Reactive power sensitivity distance $(D)$ is shown in Table 1, and the corresponding cluster tree is shown in Figure 1.

Table 1. Electrical distant between two reactive power source buses based on the sensitivity of reactive to reactive power injection.

\begin{tabular}{lcccccc}
\hline $\begin{array}{l}\text { Reactive power source } \\
\text { bus }\end{array}$ & 31 & 32 & 33 & 35 & 37 & 38 \\
\hline 31 & 0 & 0.4182 & 0.8023 & 0.7867 & 0.7069 & 0.7499 \\
32 & 0.3744 & 0 & 0.7599 & 0.7411 & 0.7028 & 0.7322 \\
33 & 0.8194 & 0.7950 & 0 & 0.4754 & 0.7545 & 0.6717 \\
35 & 0.7949 & 0.7672 & 0.4475 & 0 & 0.7211 & 0.6271 \\
37 & 0.7569 & 0.7692 & 0.7766 & 0.7591 & 0 & 0.6990 \\
38 & 0.8907 & 0.8892 & 0.8399 & 0.8274 & 0.8248 & 0 \\
\hline
\end{tabular}

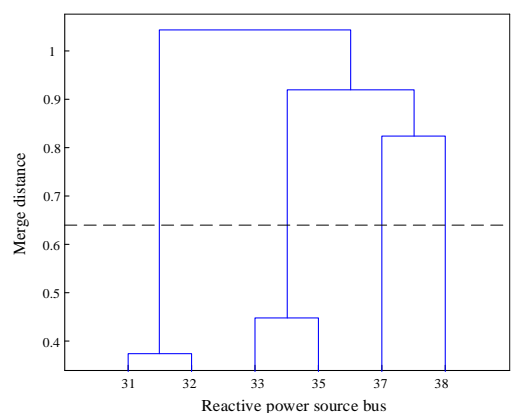

Fig. 1. Clustering result of Reactive power sources of IEEE 39-bus system.

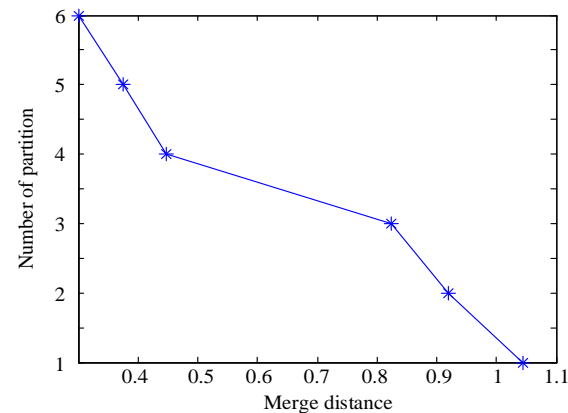

Fig. 2. Clustering curves of IEEE 39-bus system.

As shown in Figure 2, when the partition number changes from four to three, there is a relatively long platform area. And it means that the process of agglomeration from four partitions to three partitions requires a larger distance. At the same time, the slope before number four is too large. Therefore, the most appropriate and reasonable partition number is four. According to the principles described in Sec. 2.3, the load buses can be merged and the merged results are shown in Table 2.

\subsection{Secondary partition}

When system response time is unlimited, the reactive power outputs of slow sources can be fully used. So the slow sources can be regarded as fast sources. On the foundation of this, all reactive power sources can be partitioned into 6 
areas in the same processing method, and then the load buses can also be merged to each area. The final results are shown in Table 3.

Table 2. Partition results

\begin{tabular}{cc}
\hline Area number & Buses within area \\
\hline 1 & $2,25,30,37$ \\
2 & $1,3-14,31,32,39$ \\
3 & $15-24,27,33-36$ \\
4 & $26,28,29,38$ \\
\hline
\end{tabular}

Table 3. Secondary partition results.

\begin{tabular}{cc}
\hline Area number & Buses within area \\
\hline $1^{*}$ & $2,3,18,25-27,30,37$ \\
$2^{*}$ & $4-8,10-14,31,32$ \\
$3^{*}$ & $19,20,33,34$ \\
$4^{*}$ & $15-17,21-24,35,36$ \\
$5^{*}$ & $28,29,38$ \\
$6^{*}$ & $1,9,39$ \\
\hline
\end{tabular}

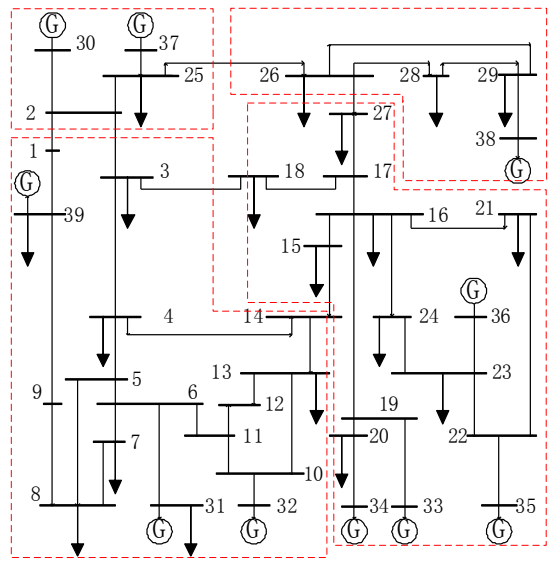

Fig. 3. Sketch map of IEEE 39-bus system with 4-area decomposition.

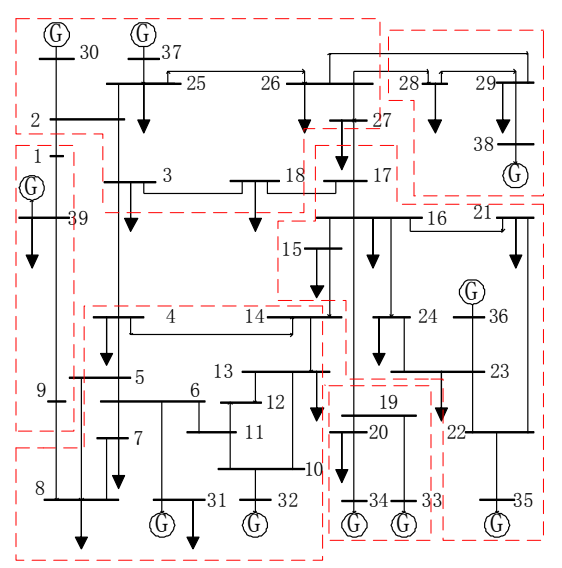

Fig. 4. Sketch map of IEEE 39-bus system. with 6-area decomposition.

\subsection{Results analysis}

By comparing the first partition results (4 areas shown in Fig.3) and secondary partition results (6 areas shown in Fig.4), there are four conclusions as follows:

(1) The area 1 of primary partition and bus $3,18,26,27$ form the new area $1^{*}$.

(2) The area 2 of primary partition is split into the new area $2^{*}$ and area $6^{*}$.

(3) The area 3 of primary partition is split into the new area $3^{*}$ and area $4^{*}$. 
(4) The bus 28, 29 and 38 form the new area $5^{*}$.

Take the bus 3 as an example, when the source 30 is considered as fast source, its reactive power compensation ability gradually works. The area 1 have source 30 and 37 to compensate reactive power together. So the control ability to bus 3 of area 1 is far beyond other areas. Meanwhile, as shown in Fig.3, the sources which most closely connected with bus 3 are source 30 and 37, and the electrical distance of the two is shortest. Based on the above analysis, the migration of bus 3 occurred.

\section{Conclusions}

Secondary partitioning based on response characteristics of reactive power sources is proposed in this paper. The bus partition of reactive power sources is built on the sensitivity of reactive power to reactive power, and then load buses are merged. By this proposed method, the reasonable partition of reactive power sources can be achieved. The response speed of reactive power sources is firstly considered in this method, and it can divide the reactive power source which is most sensitive to reactive power control of buses into the same area. Compared with other partition methods, the partition results are more reasonable and effective.

\section{References}

1. J.P. Paul, J.Y. Leost, J.M. Tesseron. Survey of the Secondary Voltage Control in France : Present Realization and Investigations. IEEE Transactions on Power Systems, vol. 2, no. 2, pp. 505-511, May. 1987.

2. P. Lagonotte, J.C. Sabonnadiere, J.Y. Leost, et al. Structural analysis of the electrical system: application to secondary voltage control in France. IEEE Power Engineering Review, vol. 4, no. 2, pp. 479-486. May. 1989.

3. J.L. Sancha, J.L. Fernandez, A. Cortes, et al. Secondary voltage control: analysis, solutions and simulation results for the Spanish transmission system. Power Industry Computer Application Conference, 1995. Conference Proceedings. 1995 IEEE. IEEE, 1995:27-32.

4. Huaichang GE, Qinglai GUO, Hongbin SUN, et al. Multivariate statistical analysis-based power-grid-partitioning method. IET Generation, Transmission \& Distribution,vol. 10,no. 4, pp. 1023-1031, Mar. 2016.

5. A.R. Brett. A Two-Stage Distributed Architecture for Voltage Control in Power Distribution Systems. IEEE Transactions on Power Systems, vol. 28, no. 2, pp. 1470-1482, Sep.2012. 
6. Xiangping NI, et.al, Effect of parameters on network partitioning schemes for power grid. Power System Technology, 2010 International Conference on, 2010, 1-6.

7. M. Benjamin, Danchi JIANG. A novel partitioning strategy for distribution networks featuring many small scale generators. Innovative Smart Grid Technologies (ISGT), 2013 IEEE PES, 2013, 1-6.

8. Yaoyu WANG, Boming ZHANG, Hongbin SUN, et al. A expert knowledge based subarea division method for hierarchical and distributed electric power system voltage/VAR optimization and control. Proceedings of the CSEE, vol. 18, no. 3, pp. 221-224, May. 1998.

9. Qinglai GUO, Hongbin SUN, Boming ZHANG, et al. Power network partitioning based on clustering analysis in Reactive power control space. Automation of Electric Power Systems, vol. 29, no. 10, pp. 36-40, May. 2005.

10. Lei FAN, Heng CHEN. Analysis of secondary voltage control: part one. Automation of Electric Power Systems, vol. 24, no. 11, pp. 18-21, May. 2000. 